\title{
Seeds of Life in Space (SOLIS)
}

\section{Formamide in protostellar shocks: Evidence for gas-phase formation}

\author{
C. Codella ${ }^{1}$, C. Ceccarelli ${ }^{2,1}$, P. Caselli ${ }^{3}$, N. Balucani ${ }^{4,1}$, V. Barone ${ }^{5}$, F. Fontani ${ }^{1}$, B. Lefloch ${ }^{2}$, L. Podio ${ }^{1}$, S. Viti ${ }^{6}$, \\ S. Feng ${ }^{3}$, R. Bachiller ${ }^{7}$, E. Bianchi ${ }^{1,8}$, F. Dulieu ${ }^{9}$, I. Jiménez-Serra ${ }^{10}$, J. Holdship ${ }^{6}$, R. Neri ${ }^{11}$, J. E. Pineda ${ }^{3}$, A. Pon ${ }^{12}$, \\ I. Sims ${ }^{13}$, S. Spezzano ${ }^{3}$, A. I. Vasyunin ${ }^{3,14}$, F. Alves ${ }^{3}$, L. Bizzocchi ${ }^{3}$, S. Bottinelli ${ }^{15,16}$, E. Caux ${ }^{15,16}$, \\ A. Chacón-Tanarro ${ }^{3}$, R. Choudhury ${ }^{3}$, A. Coutens ${ }^{6}$, C. Favre ${ }^{1,2}$, P. Hily-Blant ${ }^{2}$, C. Kahane $^{2}$, \\ A. Jaber Al-Edhari ${ }^{2,17}$, J. Laas ${ }^{3}$, A. López-Sepulcre ${ }^{11}$, J. Ospina ${ }^{2}$, Y. Oya ${ }^{18}$, A. Punanova ${ }^{3}$, C. Puzzarini ${ }^{19}$,

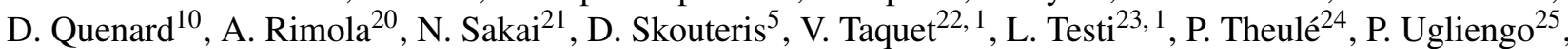 \\ C. Vastel ${ }^{15,16}$, F. Vazart ${ }^{5}$, L. Wiesenfeld ${ }^{2}$, and S. Yamamoto ${ }^{18,26}$ \\ (Affiliations can be found after the references)
}

Received 26 May 2017 / Accepted 18 August 2017

\begin{abstract}
Context. Modern versions of the Miller-Urey experiment claim that formamide $\left(\mathrm{NH}_{2} \mathrm{CHO}\right)$ could be the starting point for the formation of metabolic and genetic macromolecules. Intriguingly, formamide is indeed observed in regions forming solar-type stars and in external galaxies. Aims. How $\mathrm{NH}_{2} \mathrm{CHO}$ is formed has been a puzzle for decades: our goal is to contribute to the hotly debated question of whether formamide is mostly formed via gas-phase or grain surface chemistry.

Methods. We used the NOrthern Extended Millimeter Array (NOEMA) interferometer to image $\mathrm{NH}_{2} \mathrm{CHO}$ towards the L1157-B1 blue-shifted shock, a well-known interstellar laboratory, to study how the components of dust mantles and cores released into the gas phase triggers the formation of formamide.

Results. We report the first spatially resolved image (size $\sim 9^{\prime \prime}, \sim 2300 \mathrm{AU}$ ) of formamide emission in a shocked region around a Sun-like protostar: the line profiles are blueshifted and have a $F W H M \simeq 5 \mathrm{~km} \mathrm{~s}^{-1}$. A column density of $N_{\mathrm{NH}_{2} \mathrm{CHO}}=8 \times 10^{12} \mathrm{~cm}^{-1}$ and an abundance, with respect to $\mathrm{H}$-nuclei, of $4 \times 10^{-9}$ are derived. We show a spatial segregation of formamide with respect to other organic species. Our observations, coupled with a chemical modelling analysis, indicate that the formamide observed in L1157-B1 is formed by a gas-phase chemical process and not on grain surfaces as previously suggested.

Conclusions. The Seeds of Life in Space (SOLIS) interferometric observations of formamide provide direct evidence that this potentially crucial brick of life is efficiently formed in the gas phase around Sun-like protostars.
\end{abstract}

Key words. stars: formation - ISM: jets and outflows - ISM: molecules - ISM: individual objects: L1157-B1

\section{Introduction}

One of the main open questions in astrochemistry regards the mechanisms leading to the formation of the so-called interstellar complex organic molecules (iCOMs), which are molecules with at least six atoms; iCOMs can be considered as the building blocks of more complex pre-biotic compounds (see e.g. Caselli $\&$ Ceccarelli 2012). This topic is even more important in regions around Sun-like protostars that will produce future solarlike systems. In particular, modern versions of the Urey-Miller experiment suggest that formamide $\left(\mathrm{NH}_{2} \mathrm{CHO}\right)$ might be the starting point of metabolic and genetic species (Saladino et al. 2012). Intriguingly, formamide is detected in both Galactic highand low-mass star forming regions (e.g. Turner 1991; Nummelin et al. 1998; Halfen et al. 2011; Kahane et al. 2013; Mendoza et al. 2014; López-Sepuclre et al. 2015) and in external galaxies (Müller et al. 2013). Despite being so easily found, it is still hotly debated how this species and other iCOMs are formed (e.g. Herbst \& van Dishoeck 2009).

The two current theories predict formation by reactions in the gas phase (e.g. Vasyunin \& Herbst 2013; Balucani et al. 2015; Vasyunin et al. 2017) or on interstellar dust grains

\footnotetext{
$\star$ The reduced datacube is only available at the CDS via anonymous ftp to cdsarc.u-strasbg. fr (130.79.128.5) or via http://cdsarc.u-strasbg.fr/viz-bin/qcat?J/A+A/605/L3
}

(e.g. Garrod \& Herbst 2006; Garrod et al. 2008), the latter through surface reactions or induced by energetic processing. Focussing on formamide, the gas-phase theory proposes that it is synthesised by the reaction of formaldehyde $\left(\mathrm{H}_{2} \mathrm{CO}\right)$ and amidogen $\left(\mathrm{NH}_{2}\right)$, as suggested by Barone et al. (2015) and Vazart et al. (2016). Various mechanisms have been advanced for the formation of formamide on the grain surfaces including the combination of amidogen and formyl radical (HCO; Garrod et al. 2008; Jones et al. 2011); the hydrogenation of isocyanic acid (HNCO; Mendoza et al. 2014), the latter being most likely an inefficient reaction (Noble et al. 2015; Song et al. 2016); and particle/UV photon irradiation of ice mixtures (e.g. Kaňuchová et al. 2016; Fedoseev et al. 2016).

From an observational point of view, it is challenging to assess safely which formation mechanism dominates for formamide. The chemically rich molecular outflow driven by the L1157-mm Class 0 protostar $(d=250 \mathrm{pc})$ is a unique region that can be used to tackle this question. A precessing, episodic jet of matter at supersonic velocity emerges from L1157-mm (Gueth et al. 1996; Podio et al. 2016). The jet has excavated two main cavities, with apices called B1 and B2 (see Fig. 1). In particular, B1 consists of a series of shocks (see Sect. 3) caused by different episodes of ejection impacting against the cavity wall (Podio et al. 2016), the oldest of which (kinematical age $\simeq 1100 \mathrm{yr}$ ) is also the farthest away from the source. 
Previous observations revealed that in B1 the jet impacts caused erosion of the grain cores and ices, producing large quantities of gaseous $\mathrm{SiO}\left(\sim 10^{-7}\right.$; Gueth et al. 1998), $\mathrm{H}_{2} \mathrm{O}\left(\sim 10^{-4}\right.$; Busquet et al. 2014), and $\mathrm{HCOOCH}_{3}\left(\sim 10^{-8}\right.$; Arce et al. 2008) among other species (see also Lefloch et al. 2017). Hence, L1157-B1 provides us with a perfect place to study the reactions occurring when previously frozen species are injected into the gas, as their relative abundance evolution depends on the relative efficiency of the various reactions. Previous studies have shown that any variation on the $1000 \mathrm{AU}$ scale, such as that probed by our work, is due to the passage of shocks, rather than to differences in the composition of pre-existing, pre-shocked dust and gas (Benedettini et al. 2012; Busquet et al. 2014). To conclude, within the context of the study of iCOMs, the advantages of the L1157-B1 laboratory are twofold: (i) the source is not directly heated by the protostar, which is 0.08 pc away; and (ii) solid species in dusty icy mantles have been injected into the gas phase owing to a jet-induced shock and consequently sputtering (e.g. Bachiller et al. 2001).

L1157-B1 is one of the targets of the SOLIS ${ }^{1}$ (Seeds Of Life In Space; Ceccarelli et al. 2017, hereafter Paper III) IRAM NOrthern Extended Millimeter Array (NOEMA) large programme to investigate $\mathrm{iCOM}$ formation during the early stages of the star forming process. In this Letter we report the first high spatial resolution $\mathrm{NH}_{2} \mathrm{CHO}$ image and comparison with the acetaldehyde $\left(\mathrm{CH}_{3} \mathrm{CHO}\right)$, which allow us to constrain how gasphase chemistry matters for the formation of $\mathrm{NH}_{2} \mathrm{CHO}$.

\section{Observations}

The L1157-B1 shock was observed at $3 \mathrm{~mm}$ with the IRAM NOEMA seven-element array during several tracks in July, October, and November 2015 using both the C and D configurations. The shortest and longest baselines are $19 \mathrm{~m}$ and $237 \mathrm{~m}$, respectively, allowing us to recover emission at scales up to $\sim 17^{\prime \prime}$. The $\mathrm{NH}_{2} \mathrm{CHO}\left(4_{1,4}-3_{1,3}\right)$ line $\left(E_{\mathrm{u}}=13 \mathrm{~K}, S \mu^{2}=49 \mathrm{D}^{2}\right.$, $A_{\mathrm{ul}}=3.7 \times 10^{-5} \mathrm{~s}^{-1}$ ) at $81693.45 \mathrm{MHz}^{2}$ was observed using $80 \mathrm{MHz}$ backends with a spectral resolution of $156 \mathrm{kHz}$ $\left(\sim 0.57 \mathrm{~km} \mathrm{~s}^{-1}\right)$. We recover about $60-70 \%$ of the emission observed by Mendoza et al. (2014) using the IRAM $30 \mathrm{~m}$ (see Fig. A.1 for the $30 \mathrm{~m}$ and NOEMA spectra). Calibration was carried out following standard procedures via GILDAS-CLIC ${ }^{3}$. The bandpass was calibrated on 3C 454.3, while the absolute flux was fixed by observing MWC 349 and 0524+034; the latter was also used to set the gains in phase and amplitude. The phase rms was $\leq 50^{\circ}$, the typical precipitable water vapor (PWV) was from $10 \mathrm{~mm}$ to $40 \mathrm{~mm}$, and the system temperatures $\sim 80-100 \mathrm{~K}$ (D) and $\sim 150-250 \mathrm{~K}(\mathrm{C})$. The final uncertainty on the absolute flux scale is $\leq 15 \%$. The rms noise in the $156-\mathrm{kHz}$ channels was $2 \mathrm{mJy}$ beam $^{-1}$. Images were produced using natural weighting and restored with a clean beam of 5'.79 $\times 4^{\prime \prime} .81\left(\mathrm{PA}=-94^{\circ}\right)$.

\section{Results and discussion}

\section{1. $\mathrm{NH}_{2} \mathrm{CHO}$ spectra and maps}

Formamide emission has been detected towards L1157-B1 with a $S / N \geq 8$, confirming the $\mathrm{NH}_{2} \mathrm{CHO}$ identification by Mendoza et al. (2014) in the context of the ASAI IRAM $30 \mathrm{~m}$ spectral

\footnotetext{
1 http://solis.osug.fr/

2 Spectroscopic parameters have been extracted from the Cologne Database for Molecular Spectroscopy (Müller et al. 2005).

3 http://www. iram. fr/IRAMFR/GILDAS
}

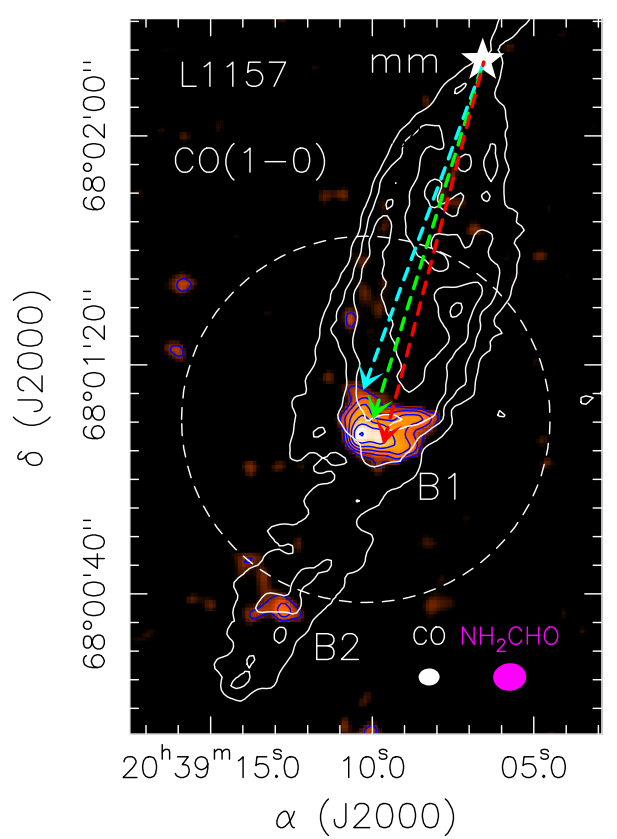

Fig. 1. Southern blue-shifted lobe L1157 in CO (1-0) (white contours; Gueth et al. 1996). The precessing jet ejected by the central object L1157-mm (white star) excavated two cavities, with apices B1 and B2, respectively. The maps are centred at $\alpha(\mathrm{J} 2000)=20^{\mathrm{h}} 39^{\mathrm{m}} 10 \mathrm{~s}$, $\delta(\mathrm{J} 2000)=+68^{\circ} 01^{\prime} 10^{\prime \prime} .5\left(\Delta \alpha=+25^{\prime \prime}\right.$ and $\Delta \delta=-63^{\prime \prime} .5$ from L1157$\mathrm{mm})$. The emission map of the $\mathrm{NH}_{2} \mathrm{CHO}\left(4_{1,4}-3_{1,3}\right)$ line (integrated over the velocity range -5 to $+5 \mathrm{~km} \mathrm{~s}^{-1}$ ) is shown by the colour image. For the $\mathrm{CO}$ image, the first contour and step are $6 \sigma(1 \sigma=$ $0.5 \mathrm{Jy} \mathrm{beam}^{-1} \mathrm{~km} \mathrm{~s}^{-1}$ ) and $4 \sigma$, respectively. The first contour and step of the $\mathrm{NH}_{2} \mathrm{CHO}$ map correspond to $3 \sigma\left(15 \mathrm{mJy}\right.$ beam $\left.{ }^{-1} \mathrm{~km} \mathrm{~s}^{-1}\right)$ and $1 \sigma$, respectively. The dashed circle indicates the primary beam of the $\mathrm{NH}_{2} \mathrm{CHO}$ image (64"). The magenta and white ellipses depict the synthesised beams of the $\mathrm{NH}_{2} \mathrm{CHO}\left(5^{\prime \prime} \cdot 79 \times 44^{\prime \prime} 81, \mathrm{PA}=-94^{\circ}\right)$ and $\mathrm{CO}$ $\left(3^{\prime \prime} 65 \times 2^{\prime \prime}\right.$ ' $\left.96, \mathrm{PA}=+88^{\circ}\right)$ observations, respectively. The three dashed arrows indicate the directions (projected on the plane of the sky) of the episodic jet producing the shocks analysed in Sect. 3 and Fig. 2.

survey. Figure 1 shows the map of the $\mathrm{NH}_{2} \mathrm{CHO}\left(4_{1,4}-3_{1,3}\right)$ integrated emission on top of the CO (1-0) image (Gueth et al. 1996), which well outlines the B1 and B2 cavities opened by the precessing jet driven by the L1157-mm protostar, located (in Fig. 1) at $\Delta \alpha=-25^{\prime \prime}$ and $\Delta \delta=+63$ '.5. Formamide is emitted from an extended region with a beam deconvolved size of $\simeq 9^{\prime \prime}$ ( 2300 AU), which is clearly associated with the apex of the B1 cavity. In addition, weaker $(S / N \geq 4)$ emission appears in correspondence with the older B2 peak; however, this peak is affected by primary-beam attenuation because it is more than $30^{\prime \prime}$ from B1. For that reason, further analysis will be focussed on the B1 region. The line at the peak emission (see Fig. A.1) has a linewidth with a full width half maximum (FWHM) of $4.6 \pm 0.6 \mathrm{~km} \mathrm{~s}^{-1}$ and peaks close to $\sim 0 \mathrm{~km} \mathrm{~s}^{-1}$, thus it is blueshifted $\left(v_{\text {sys }}=+2.6 \mathrm{~km} \mathrm{~s}^{-1}\right.$; e.g. Bachiller et al. 2001). Using the emitting size and assuming optically thin conditions and an excitation temperature of $10 \mathrm{~K}$, as derived by several formamide lines observed with the IRAM $30 \mathrm{~m}$ antenna, (Mendoza et al. 2014), the average formamide column density is $N_{\mathrm{NH}_{2} \mathrm{CHO}}=$ $8 \times 10^{12} \mathrm{~cm}^{-1}$. This corresponds to an estimated average abundance (with respect to $\mathrm{H}$-nuclei) of about $4 \times 10^{-9}$, assuming a $\mathrm{H}$ column density of $2 \times 10^{21} \mathrm{~cm}^{-2}$ derived for the cavity by Lefloch et al. (2012). 


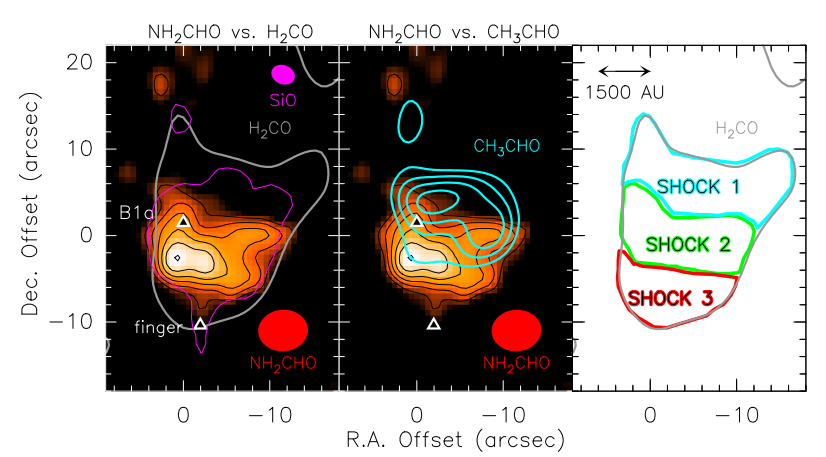

Fig. 2. Chemical segregation in L1157-B1. The maps are centred at $\alpha(\mathrm{J} 2000)=20^{\mathrm{h}} 39^{\mathrm{m}} 09 \mathrm{~s}, \delta(\mathrm{J} 2000)=+68^{\circ} 01^{\prime} 10^{\prime \prime} .0$. Left panel: $\mathrm{p}-$ $\mathrm{H}_{2} \mathrm{CO}\left(2_{0,2}-1_{0,1}\right)$ integrated emission (grey; Benedettini et al. 2013), and $\mathrm{SiO}(2-1)$ (magenta) at low velocity (less than $-10 \mathrm{~km} \mathrm{~s}^{-1}$ with respect to $v_{\text {sys }}$; Gueth et al. 1998) on top of the present $\mathrm{NH}_{2} \mathrm{CHO}$ line emission map (colour image, black contours). For clarity, for the $\mathrm{H}_{2} \mathrm{CO}$ and $\mathrm{SiO}$ images, only the $3 \sigma\left(1 \sigma=3.3 \mathrm{mJy} \mathrm{beam}^{-1} \mathrm{~km} \mathrm{~s}^{-1}\right.$ and $50 \mathrm{mJy}$ beam ${ }^{-1} \mathrm{~km} \mathrm{~s}^{-1}$ for $\mathrm{H}_{2} \mathrm{CO}$ and $\mathrm{SiO}$, respectively) contour is reported to show the overall B1 structure (see Fig. A.2 for the complete set of contours). The northern triangle at $\mathrm{B} 1 \mathrm{a}$ identifies the youngest position in B1, where the precessing jet driven by L1157-mm impacts the cavity wall (Gueth et al. 1998; Busquet et al. 2014), while the southern triangle denotes the position of the oldest shock, which is identified by the so-called "finger" feature traced by $\mathrm{SiO}$ at low velocity. The $\mathrm{H}_{2} \mathrm{CO}$ image is smoothed to the same angular resolution (red ellipse; see Fig. 1) of the $\mathrm{NH}_{2} \mathrm{CHO}\left(4_{1,4}-3_{1,3}\right)$ line data. The synthesised beam of the $\mathrm{SiO}$ is $2^{\prime \prime} \cdot 8 \times 22^{\prime \prime} 2, \mathrm{PA}=56^{\circ}$. Middle panel: same as in the left panel for the $\mathrm{CH}_{3} \mathrm{CHO}\left(7_{0,7}-6_{0,6} \mathrm{E}+\mathrm{A}\right)$ velocity-integrated emission (cyan contours; also smoothed to the same beam of the $\mathrm{NH}_{2} \mathrm{CHO}$ map; first contour and step correspond to $3 \sigma, 4 \mathrm{mJy} \mathrm{beam}^{-1} \mathrm{~km} \mathrm{~s}^{-1}$, and $1 \sigma$, respectively; Codella et al. 2015). Right panel: sketch of the three zones identified from the spatial distribution of formamide and acetaldehyde: SHOCK 1 (blue), the northern region, where $\mathrm{CH}_{3} \mathrm{CHO}$ (and not $\mathrm{NH}_{2} \mathrm{CHO}$ ) is detected; SHOCK 2 (green), where both $\mathrm{CH}_{3} \mathrm{CHO}$ and $\mathrm{NH}_{2} \mathrm{CHO}$ are detected; and SHOCK 3 (red), the southern region, where only $\mathrm{NH}_{2} \mathrm{CHO}$ (and not $\mathrm{CH}_{3} \mathrm{CHO}$ ) is detected. Time increases and chemistry evolves going from SHOCK 1 to SHOCK 3 (see dashed arrows in Fig. 1).

\subsection{Formamide and acetaldehyde spatial anticorrelation}

In Fig. 2, we report a zoom-in of the B1 structure, as traced by the line emission from formaldehyde and $\mathrm{SiO}$ (Gueth et al. 1998; Benedettini et al. 2013). The figure clearly shows the first important result of these observations: the formamide emission does not coincide with that from $\mathrm{H}_{2} \mathrm{CO}$ and $\mathrm{SiO}$, but only covers the southern portion of the B1 structure. Also, Fig. 2 reports the emission from another iCOM, acetaldehyde $\left(\mathrm{CH}_{3} \mathrm{CHO}\right.$; Codella et al. 2015). Surprisingly, unlike formamide, it is mostly associated with the northern portion of B1. When the difference between these two species is considered, one can identify three zones as follows: SHOCK 1: the northern and youngest zone, where only acetaldehyde emits $\left(X\left(\mathrm{CH}_{3} \mathrm{CHO}\right) / X\left(\mathrm{NH}_{2} \mathrm{CHO}\right)\right.$ abundance ratio $>8$; see Fig. 3$)$; SHOCK 2: an intermediate zone, where both formamide and acetaldehyde are present $\left(X\left(\mathrm{CH}_{3} \mathrm{CHO}\right) / X\left(\mathrm{NH}_{2} \mathrm{CHO}\right)=2-8\right)$; and SHOCK 3: the southern and oldest region, where only formamide emits $\left(X\left(\mathrm{CH}_{3} \mathrm{CHO}\right) / X\left(\mathrm{NH}_{2} \mathrm{CHO}\right)<2\right)$.

The analysis of the $\mathrm{SiO}$ and HDCO distribution (Fig. A.2) confirms that B1 is composed by at least two different shocks and is not a single bow-like shock. Specifically, (A) the northern part, SHOCK 1, is associated with the youngest shock (within the $\mathrm{B} 1$ structure) at B1a, which is characterised by (i) the emission of HDCO, which is a selective tracer of dust mantle release

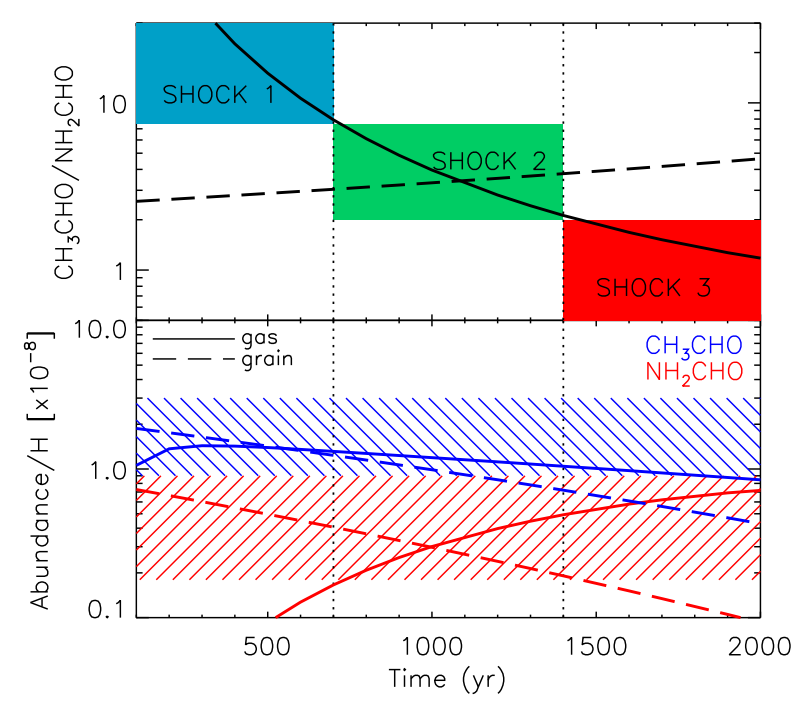

Fig. 3. Model predictions of acetaldehyde and formamide after a shock passage. Upper panel: $\mathrm{CH}_{3} \mathrm{CHO} / \mathrm{NH}_{2} \mathrm{CHO}$ calculated abundance ratio as a function of time from the passage of the shock. Solid lines refer to a model in which acetaldehyde and formamide are both synthesised in the gas phase, whereas dashed lines refer to predictions assuming that they are injected into the gas phase directly from the grain mantles. The vertical ranges of the three coloured boxes represent the measured ranges, including the uncertainties of the $\mathrm{CH}_{3} \mathrm{CHO} / \mathrm{NH}_{2} \mathrm{CHO}$ abundance ratio towards the three zones identified in Fig. 2: SHOCK 1 (ratio >8), SHOCK 2 (ratio 2-8), and SHOCK 3 (ratio $\leq 2$ ). When not detected, we derived the upper limits on $\mathrm{CH}_{3} \mathrm{CHO}$ and $\mathrm{NH}_{2} \mathrm{CHO}$ with the $3 \sigma$ value. The two dotted vertical lines define the time when the formamide and acetaldehyde abundance ratios (as derived by the gas-phase model) fall below the minimum measured values. Lower panel: acetaldehyde $\left(\mathrm{CH}_{3} \mathrm{CHO}\right.$, blue $)$ and formamide $\left(\mathrm{NH}_{2} \mathrm{CHO}\right.$, red) abundances, with respect to $\mathrm{H}$-nuclei, as a function of time from the passage of the shock. The dashed blue and red regions show the maximum and minimum $\mathrm{CH}_{3} \mathrm{CHO}$ and $\mathrm{NH}_{2} \mathrm{CHO}$ measured abundances.

(Fontani et al. 2014), and (ii) extremely high-velocity SiO emission that traces the current sputtering of the dust refractory cores. (B) The southern region, SHOCK 3 is associated with the oldest shock (within B1) because (i) no HDCO is observed, and (ii) $\mathrm{SiO}$ emission is only observed at low velocity and shows a "finger" pointing south (Gueth et al. 1998). This implies that either $\mathrm{SiO}$ molecules have been slowed down with time with respect to the high velocities (needed to produce gaseous $\mathrm{Si}$ ) or that the shock incident angle has changed, so that the projected velocity is lower. In both cases this indicates that a unique shock structure for L1157-B1 is ruled out. (C) The central region, between SHOCK 1 and SHOCK 3, is characterised by the occurrence of the bulk of the low-velocity $\mathrm{SiO}$ molecules, which, once produced at high velocities, have plausibly slowed down with time. It is then reasonable to assume that this region is associated with a third, intermediate in time, shock event. However, the results and conclusions of the present paper are based on the occurrence of at least two shocks of different age (SHOCKs 1 and 3).

We notice that the difference in the three zones cannot be attributed to excitation effects, as the mapped formamide and acetaldehyde lines have similar upper level energies $(11 \mathrm{~K}$ and $26 \mathrm{~K})$, similar Einstein coefficients $\left(\sim 10^{-5} \mathrm{~s}^{-1}\right)$, and the derived excitation temperatures are also similar (i.e. $10 \mathrm{~K}$ against $15 \mathrm{~K}$, for formamide and acetaldehyde respectively; Mendoza et al. 2014; Codella et al. 2015). Besides, there is no evidence of a monotonic volume density gradient across the $\mathrm{B} 1$ region from north to south (Benedettini et al. 2013; Gómez-Ruiz et al. 2015), 
as in the case of $\mathrm{CH}_{3} \mathrm{CHO} / \mathrm{NH}_{2} \mathrm{CHO}$ line intensity ratio (see Fig. A.2). Therefore, the difference between the three zones must be due to a difference in the chemical composition, thus indicating a clear evolutionary effect.

\section{Chemical modelling}

To understand what the observed chemical differentiation implies, we ran an astrochemical model (a modified version of Nahoon, Loison et al. 2014, see Appendix B) considering three possibilities: (i) formamide and acetaldehyde are grain-surface chemistry products, (ii) formamide and acetaldehyde are gasphase chemistry products, and (iii) one of the two species is a grain-surface and the other one a gas-phase chemistry product. Briefly, we use a time-dependent gas-phase code that follows the chemical evolution of the gas. This code starts with the chemical composition of a molecular cloud and then simulates the passage of the shock by suddenly increasing the gas density and temperature to $10^{5} \mathrm{~cm}^{-3}$ and $60 \mathrm{~K}$, respectively, (i.e. the typical values measured for the B1 cavities, 20-80 K; Lefloch et al. 2012; 0.5$10 \times 10^{5} \mathrm{~cm}^{-3}$; Gómez-Ruiz et al. 2015) and the gaseous abundance of grain mantle molecules. The abundances of the mantle molecules are assumed to be similar to those measured by IR observations of the dust ices (Boogert et al. 2005) or specifically constrained by previous studies on L1157-B1 (see Table B.1). The chemical network is described in Appendix B. The results of the modelling are discussed for the three cases mentioned above. 1. Grain-surface formation of $\mathrm{CH}_{3} \mathrm{CHO}$ and $\mathrm{NH}_{2} \mathrm{CHO}$ : First, we assume that both formamide and acetaldehyde are synthesised on the grain surfaces and that the passage of the shock injects these two species into the gas phase in quantities such that the measured abundances are roughly reproduced. The predicted abundances as a function of time are shown in Fig. 3. They decrease by approximately the same factor in a $2000 \mathrm{yr}$ interval. Actually, the predicted $\left[\mathrm{CH}_{3} \mathrm{CHO}\right] /\left[\mathrm{NH}_{2} \mathrm{CHO}\right]$ abundance ratio slightly increases with time, which is in contrast with the observations that show exactly the opposite trend. Therefore, the pure grain-surface hypothesis cannot explain the observed formamide/acetaldehyde segregation. In other words, our observations rule out the hypothesis that the bulk of the observed acetaldehyde and formamide in L1157-B1 are both directly injected from the grain mantles into the gas phase.

2. Gas-phase formation of $\mathrm{CH}_{3} \mathrm{CHO}$ and $\mathrm{NH}_{2} \mathrm{CHO}$ : We then assumed that both acetaldehyde and formamide are formed in the gas phase from species previously on the grain mantles and injected into the gas phase during the shock passage. Acetaldehyde is assumed to be formed by the reaction of ethyl radical $\left(\mathrm{CH}_{3} \mathrm{CH}_{2}\right)$ with atomic oxygen (Charnley et al. 2004): $\mathrm{CH}_{3} \mathrm{CH}_{2}+\mathrm{O} \rightarrow \mathrm{CH}_{3} \mathrm{CHO}+\mathrm{H}$. Formamide is assumed to be formed by the reaction of amidogen with formaldehyde (Barone et al. 2015; Vazart et al. 2016): $\mathrm{NH}_{2}+\mathrm{H}_{2} \mathrm{CO} \rightarrow \mathrm{NH}_{2} \mathrm{CHO}+$ $\mathrm{H}$. We ran various models with different values of ethyl radical, ammonia (mother of $\mathrm{NH}_{2}$ ), and formaldehyde, to reproduce the observed abundances. We also ran alternative tests injecting ethane $\left(\mathrm{CH}_{3} \mathrm{CH}_{3}\right)$, the fully hydrogenated so-called cousin of ethyl radical, and amidogen, a partially hydrogenated cousin of ammonia, into the gas. The best agreement with observations is obtained by injecting into the gas phase $4 \times 10^{-8}$ of ethyl radical, $2 \times 10^{-5}$ of ammonia, and $1 \times 10^{-6}$ of formaldehyde (see Appendix B for details). This model not only reproduces fairly well the observed abundances (see Fig. 3), it also fits the behaviour of the $\left[\mathrm{CH}_{3} \mathrm{CHO}\right] /\left[\mathrm{NH}_{2} \mathrm{CHO}\right]$ abundance ratio, where acetaldehyde is more abundant in the younger northern SHOCK 1 and formamide is more abundant in the older southern SHOCK 3. The evolution timescale is sensitive to the cosmic ray ionisation rate $\zeta$. We find that the best agreement with the observations is obtained when $\zeta$ is $6 \times 10^{-16} \mathrm{~s}^{-1}$, which is very close to that previously found (Podio et al. 2014) based on the analysis of the molecular ions in L1157-B1. Finally, a larger shocked gas density would result in speeding up the chemical evolution. As a consequence, the $\mathrm{CH}_{3} \mathrm{CHO} / \mathrm{NH}_{2} \mathrm{CHO}$ abundance ratio curve would be shifted towards earlier times. For example, if the density were 10 times larger, namely $2 \times 10^{6} \mathrm{~cm}^{-3}$, the curve would be shifted earlier by about $1000 \mathrm{yr}$. This just means that a substantial difference, by a factor 10 , in the gas density at SHOCKs 1 and 3 would not change our major conclusions, but would just imply a slightly smaller cosmic ray ionisation rate.

3. Either acetaldehyde or formamide is a grain-surface and the other a gas-phase chemistry product: We checked the possibility that acetaldehyde is synthesised on the grain surfaces and formamide in the gas and we obtained results similar to the case (2). Hence, the gaseous $\mathrm{CH}_{3} \mathrm{CHO}$ abundance evolution is rather independent on the formation route (surface chemistry or gas-phase chemistry). We finally checked the possibility that the gas-phase reaction $\mathrm{NH}_{2}+\mathrm{H}_{2} \mathrm{CO}$ is not efficient (Song \& Kästner 2016). In this case, no model can reproduce the observations (both the abundance and the evolution).

In summary, the new SOLIS observations indicate that the formation of observed formamide in L1157-B1 is dominated by gas-phase reactions involving species previously hydrogenated on the grain surfaces, although we cannot exclude a minor contribution from mechanisms such as energetic processing of ices. The formamide abundance needs to peak when the acetaldehyde abundance has already started to decrease. This is only possible if formamide is mostly formed in the gas phase and the reaction between amidogen and formaldehyde (Barone et al. 2015; Vazart et al. 2016) successfully reproduces the observations. Although simple, our model catches the essential aspects of the chemical behaviour of formamide and acetaldehyde, namely their abundance as a function of time once the shock has passed. Indeed, the major uncertainties lie in the used chemical network more than in the detailed physical processes or the detailed gasgrain interactions (see Appendix B for more). In this context, it is encouraging that the age difference between SHOCK 1 and SHOCK 3 derived by our simple astrochemical model $(\sim 700 \mathrm{yr})$ is the same order of magnitude of the one $(\sim 2000 \mathrm{yr})$ independently derived by dynamical studies of L1157-B1 (Podio et al. 2016). A more detailed modelling including a more complex and realistic treatment of the shock will be necessary to confirm that this is not just a coincidence and to refine the present predictions.

\section{Conclusions}

The present work demonstrates that the formamide observed in L1157-B1 is dominated by gas-phase chemistry and that the reaction $\mathrm{NH}_{2}+\mathrm{H}_{2} \mathrm{CO} \rightarrow \mathrm{NH}_{2} \mathrm{CHO}+\mathrm{H}$ explains the observations. Although we are unable to place constraints on the acetaldehyde formation route, we note that quantum chemistry computations have shown that the simple combination of the methyl radical $\left(\mathrm{CH}_{3}\right)$ and formyl radical (HCO) is an inefficient channel on water ice surfaces (Enrique-Romero et al. 2016), so that it is possible that $\mathrm{CH}_{3} \mathrm{CHO}$ is also a gas-phase product. The recent detection of iCOMs in cold objects (e.g. Vastel et al. 2014) has already challenged a pure grain-surface chemistry paradigm for their formation (e.g. Vasyunin \& Herbst 2013, and references therein). These new observations add evidence that gas-phase chemistry plays an important role in the game of iCOM formation. 
Acknowledgements. We are very grateful to all the IRAM staff, whose dedication allowed us to carry out the SOLIS project. We also thank the anonymous referee for useful suggestions. This work was supported by (i) the French programme "Physique et Chimie du Milieu Interstellaire" (PCMI) funded by the Conseil National de la Recherche Scientifique (CNRS) and Centre National d'Études Spatiales (CNES), (ii) by the Italian Ministero dell'Istruzione, Università e Ricerca through the grant Progetti Premiali 2012 - iALMA (CUP C52I13000140001), (iii) by the programme PRIN-MIUR 2015 STARS in the CAOS - Simulation Tools for Astrochemical Reactivity and Spectroscopy in the Cyberinfrastructure for Astrochemical Organic Species (2015F59J3R, MIUR Ministero dell'Istruzione, dell'Università della Ricerca e della Scuola Normale Superiore), and (iv) by the French Agence Nationale de la Recherche (ANR), under reference ANR-12-JS05-0005. P.C., A.C.T., J.E.P., and A.P.u. acknowledge support from the European Research Council (ERC; project PALs 320620). A.P.o. acknowledges the financial support provided by a Canadian Institute for Theoretical Astrophysics (CITA) National Fellowship. I.J.-S. and D.Q. acknowledge the financial support received from the STFC though an Ernest Rutherford Fellowship (proposal number ST/L004801) and Grant (ST/M004139).

\section{References}

Arce, H. G., Santiago-García, J., Jørgensen, J. K., Tafalla, M., \& Bachiller, R. 2008, ApJ, 681, L21

Bachiller, R., Peréz Gutiérrez, M., Kumar, M. S. N., \& Tafalla, M. 2001, A\&A, 372,899

Balucani, N., Ceccarelli, C., \& Taquet, V. 2015, MNRAS, 449, L16

Barone, V., Latouche, C., Skouteri, D., et al. 2015, MNRAS, 453, L31

Benedettini, M., Busquet, G., Lefloch, B., et al. 2012, A\&A, 539, L3

Benedettini, M., Viti, S., Codella, C., et al. 2013, MNRAS, 436, 179

Boogert, A. C. A., Gerakines, P. A., \& Whittet, D. C.B. 2015, ARA\&A, 53, 541

Busquet, G., Lefloch, B., Benedettini, M., et al. 2014, A\&A, 561, A120

Caselli, P., \& Ceccarelli, C. 2012, A\&ARv, 20, 56

Ceccarelli, C., Caselli, P., Fontani, F., et al. 2017, ApJ, submitted

Charnley, S. B., Tielens, A. G. G. M., \& Millar, T. J. 1992, ApJ, 399, L71

Codella, C., Fontani, F., Ceccarelli, C., et al. 2015, MNRAS, 449, L11

Enrique-Romero, J., Rimola, A., Ceccarelli, C., \& Balucani, N. 2016, MNRAS, 459, L8

Fedoseev, G., Chuang, K.-J., van Dishoeck, E. F., Ioppolo, S., \& Linnartz, H. 2016, MNRAS, 460, 4297

Fontani, F., Codella, C., Ceccarelli, C., et al. 2014, ApJ, 788, L43

Garrod, R. T., \& Herbst, E. 2006, A\&A, 457, 927

Garrod, R. T., Widicus Weaver, S. L., \& Herbst, E. 2008, ApJ, 682, 283

Gómez-Ruiz, A. I., Codella, C., Lefloch, B., et al. 2015, MNRAS, 446, 3346

Gueth, F., Guilloteau, S., \& Bachiller, R. 1996, A\&A, 307, 891

Gueth, F., Guilloteau, S., \& Bachiller, R. 1998, A\&A, 333, 287

Hamberg, M., Zhaunerchyk, V., Vigren, E., et al. 2010, A\&A, 522, A90

Halfen, D. T., Ilyushin, V., \& Ziurys, L. M. 2011, ApJ, 743, 60

Harding, L. B., Klippenstein, S. J., \& Georgievskii, Y. 2005, Proc. Combust. Inst., 30, 985

Herbst, E., \& van Dishoeck, E. F. 2009, ARA\&A, 47, 427

Jones, B. M., Bennett, C. J., \& Kaiser, R. I. 2011, ApJ, 734, 78

Kahane, C., Ceccarelli, C., Faure, A., \& Caux, E. 2013, ApJ, 763, L38

Kaňuchová, Z., Urso, R. G., Baratta, G. A., et al. 2016, A\&A, 585, A155

Lefloch, B., Cabrit, S., Busquet, G., et al. 2012, ApJ, 757, L25

Lefloch, B., Ceccarelli, C., Codella, C., et al. 2017, MNRAS, 469, L73

Loison, J.-C., Wakelam, V., Hickson, K. M., Bergeat, A., \& Mereau, R. 2014 MNRAS, 437, L930

López-Sepulcre, A., Jaber, A. A., Mendoza, E., et al. 2015, MNRAS, 449, 2438

Mendoza, E., Lefloch, B., López-Sepulcre, A., et al. 2014, MNRAS, 445, 151

Müller, H. S. P., Schöier, F. L., Stutzki, J., \& Winnewisser, G. 2005, J. Mol. Struct., 742, 215

Müller, S., Beelen, A., Black, J. H., et al. 2013, A\&A, 551, A109

Noble, J. A., Theule, P., Congiu, E., et al. 2015, A\&A, 576, A91

Nummelin, A., Bergman, P., Hjalmarson, A., et al. 1998, ApJS, 117, 427

Podio, L., Lefloch, B., Ceccarelli, C., Codella, C., \& Bachiller, R. 2014, A\&A, 565, A64

Podio, L., Codella, C., Gueth, F., et al. 2016, A\&A, 593, L4

Saladino, R., Botta, G., Pino, S., Costanzo, G., \& Di Mauro, E. 2012, Chem. Soc. Rev., 41, 5526
Skouteris, D., Vazart, F., Ceccarelli, C., et al. 2017, MNRAS, 468, L1 Song, L., \& Kästner, J. 2016, J. Phys. Chem. Chem. Phys., 18, 29278 Tafalla, M., \& Bachiller, R. 1995, ApJ, 443, L37

Turner, B. E. 1991, ApJS, 76, 617

Vastel, C., Ceccarelli, C., Lefloch, B., \& Bachiller, R. 2014, MNRAS, 795, L2 Vasyunin, A. I., \& Herbst, E. 2013, ApJ, 769, 34

Vasyunin, A. I., Caselli, P., Dulieu, F., \& Jiménez-Serra, I. 2017, ApJ, 842, 33

Vazart, F., Calderini, D., Puzzarini, C., Skouteris, D., \& Barone, V. 2016, J. Chem. Theory, Comput., 12, 5385

Yang, Y., Zhang, W. J., Gao, X. M., et al. 2005, Chin. J. Chem. Phys., 18, 515

1 INAF, Osservatorio Astrofisico di Arcetri, Largo E. Fermi 5, 50125 Firenze, Italy e-mail: codella@arcetri.astro.it

2 Univ. Grenoble Alpes, CNRS, Institut de Planétologie et d'Astrophysique de Grenoble (IPAG), 38000 Grenoble, France

3 Max-Planck-Institut für extraterrestrische Physik (MPE), Giessenbachstrasse 1, 85748 Garching, Germany

4 Dipartimento di Chimica, Biologia e Biotecnologie, via Elce di Sotto 8, 06123 Perugia, Italy

5 Scuola Normale Superiore, Piazza dei Cavalieri 7, 56126 Pisa, Italy

6 Department of Physics and Astronomy, University College London, Gower Street, London, WC1E 6BT, UK

7 IGN, Observatorio Astronómico Nacional, Calle Alfonso XII, 28004 Madrid, Spain

8 Dipartimento di Fisica e Astronomia, Università degli Studi di Firenze, Italy

9 LERMA, Université de Cergy-Pontoise, Observatoire de Paris, PSL Research University, CNRS, Sorbonne Université, UPMC, Univ. Paris 06, 95000 Cergy Pontoise, France

10 School of Physics and Astronomy Queen Mary, University of London, 327 Mile End Road, E1 4NS London, UK

11 Institut de Radioastronomie Millimétrique, 300 rue de la Piscine, Domaine Universitaire de Grenoble, 38406 Saint-Martin d'Hères, France

12 Department of Physics \& Astronomy, The University of Western Ontario, 1151 Richmond Street, London, Ontario, N6A 3K7, Canada

13 Université de Rennes 1, Institut de Physique de Rennes, Rennes, France

14 Ural Federal University, Ekaterinburg, Russia

15 Université de Toulouse, UPS-OMP, IRAP, Toulouse, France

16 CNRS, IRAP, 9 Av. Colonel Roche, BP 44346, 31028 Toulouse Cedex 4, France

17 University of AL-Muthanna, College of Science, Physics Department, Al-Muthanna, Iraq

18 Department of Physics, The University of Tokyo, 7-3-1, Hongo, Bunkyo-ku, Tokyo 113-0033, Japan

19 Dipartimento di Chimica "Giacomo Ciamician", via F. Selmi, 2, 40126 Bologna, Italy

20 Departament de Química, Universitat Autònoma de Barcelona, 08193 Bellaterra, Catalonia, Spain

21 The Institute of Physical and Chemical Research (RIKEN), 2-1, Hirosawa, Wako-shi, Saitama 351-0198, Japan

22 Leiden Observatory, Leiden University, 9513, 2300 RA Leiden, The Netherlands

23 ESO, Karl Schwarzchild Srt. 2, 85478 Garching bei München, Germany

24 Aix-Marseille Université, PIIM UMR-CNRS 7345, 13397 Marseille, France

25 Università degli Studi di Torino, Dipartimento Chimica via Pietro Giuria 7, 10125 Torino, Italy

26 Research Center for the Early Universe, The University of Tokyo, 7-3-1, Hongo, Bunkyo-ku, Tokyo 113-0033, Japan 


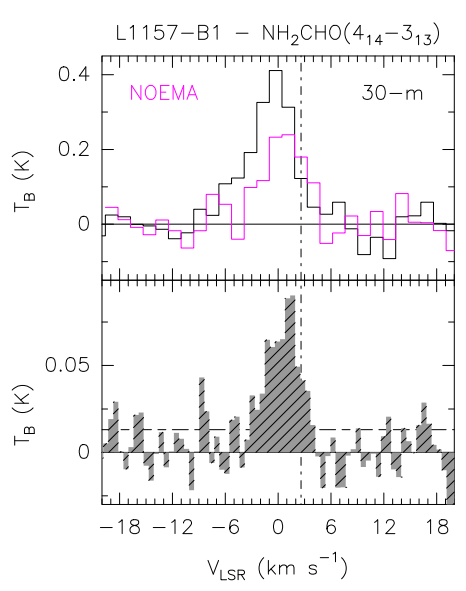

Fig. A.1. Upper panel: comparison in $T_{\mathrm{B}}$ scale of the $\mathrm{NH}_{2} \mathrm{CHO}\left(4_{1,4}-\right.$ $3_{1,3}$ ) spectrum as observed using the IRAM $30 \mathrm{~m}$ antenna (Mendoza et al. 2014) and that extracted from the present NOEMA map from a circular region equal to the IRAM $30 \mathrm{~m}$ HPBW $\left(30^{\prime \prime}\right) . F_{v}(\mathrm{Jy})=$ $4.9493 T_{\mathrm{B}}(\mathrm{K})$. The NOEMA spectrum has been smoothed to match the IRAM $30 \mathrm{~m}$ velocity resolution. Lower panel: emission (in $T_{\mathrm{B}}$ scale) extracted at the peak of the formamide spatial distribution (see Fig. 2). Horizontal dashed line indicates the $1 \sigma$ noise level $(13 \mathrm{mK})$.

\section{Appendix A: Additional line spectra and maps}

Figure A.1 (upper panel) shows the comparison in flux density scale between the $\mathrm{NH}_{2} \mathrm{CHO}\left(4_{1,4}-3_{1,3}\right)$ spectrum as observed using the IRAM $30 \mathrm{~m}$ antenna (Mendoza et al. 2014) and that extracted from the present NOEMA map from a circular region equal to the IRAM $30 \mathrm{~m}$ half power beam width (HPBW) of $30^{\prime \prime}$. The lines are blue-shifted $\left(v_{\text {sys }}=+2.6 \mathrm{~km} \mathrm{~s}^{-1}\right.$; Bachiller et al. 2001). Between $60 \%$ and $70 \%$ of the emission observed using the IRAM single dish is recovered by the NOEMA interferometer, which filters out emission structures larger than $17^{\prime \prime}$. Figure A.1 (lower panel) also shows the $\mathrm{NH}_{2} \mathrm{CHO}\left(4_{1,4}-3_{1,3}\right)$ emission line (in brightness temperature scale, $T_{\mathrm{B}}$ ) observed at the peak of the formamide spatial distribution (Figs. 1, 2).

Figure A. 2 shows how different shocks are present within the L1157-B1 structure. The northern region (see the B1a position) is associated with both $\mathrm{SiO}$ emitting very high velocities (up to $-18 \mathrm{~km} \mathrm{~s}^{-1}$ with respect to $v_{\text {sys }}$ ), as well as with HDCO, which is a selective tracer of dust mantle release. On the other hand, the southern region is characterised by no HDCO and by lowvelocity $\mathrm{SiO}$ emission producing the so-called "finger" pointing towards south (Gueth et al. 1998).

\section{Appendix B: Sensitivity to the model parameters}

In order to understand the origin of the observed spatial segregation between the acetaldehyde and formamide emission, we ran a chemical model with the aim to simulate the passage of the shock. To this end, we used a modified (to make it more flexible) version of Nahoon (Loison et al. 2014), and a chemical network consisting of 511 species and 7792 reactions. The base of the chemical network is KIDA.2014 ${ }^{4}$, which has been augmented and corrected with updated reactions (Loison et al. 2014; Balucani et al. 2015; Barone et al. 2015). To simulate the passage of the shock, we followed the strategy used in previous works (Podio et al. 2014; Codella et al. 2015), namely a twostep modelling. In the first step, we ran a model assuming the conditions of the gas before the passage of the shock, namely a gas cloud of $2 \times 10^{4} \mathrm{H}$-nuclei $\mathrm{cm}^{-3}$ and a temperature of $10 \mathrm{~K}$.

\footnotetext{
4 http://kida.obs.u-bordeaux1.fr
}

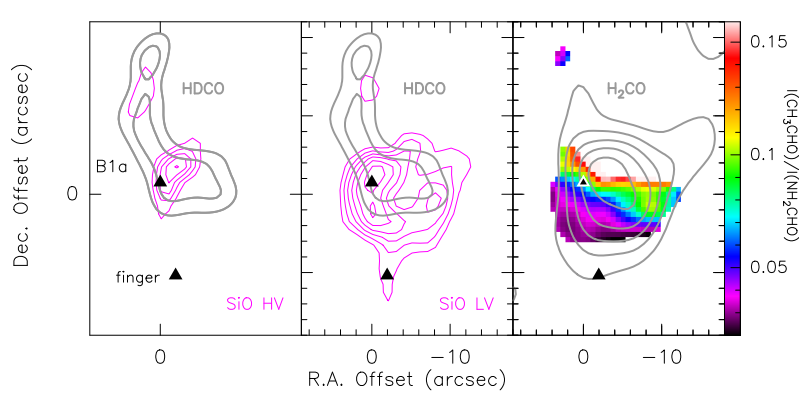

Fig. A.2. Different shocks in L1157-B1, as traced by HDCO and SiO high- and low-velocity emission. The maps are centred as in Fig. 2. The beams of all the images are reported in Figs. 1 and 2. Left panel: HDCO $\left(2_{1,1}-1_{0,1}\right)$ integrated emission (grey contours; smoothed to the same beam of the $\mathrm{NH}_{2} \mathrm{CHO}$ map; Fontani et al. 2014) tracing the dust mantle release due to the youngest (within B1) impact (the B1a position; northern black triangle) of the jet against the cavity wall (Gueth et al. 1998; Busquet et al. 2014). The magenta contours represent the high-velocity ( $\mathrm{HV}$; from $-10 \mathrm{~km} \mathrm{~s}^{-1}$ up to $-18 \mathrm{~km} \mathrm{~s}^{-1}$ with respect to $\left.v_{\text {sys }}\right) \mathrm{SiO}(2-1)$ emission, tracing a smaller region associated with the release of $\mathrm{SiO}$ from the dust refractory core. For the HDCO image, the first contour and step are $3 \sigma\left(1 \sigma=1 \mathrm{mJy} \mathrm{beam}^{-1} \mathrm{~km} \mathrm{~s}^{-1}\right)$ and $1 \sigma$, respectively. For $\mathrm{SiO}$ at $\mathrm{HV}$, the first contour and step are $5 \sigma$ $\left(1 \sigma=27 \mathrm{mJy} \mathrm{beam}^{-1} \mathrm{~km} \mathrm{~s}^{-1}\right)$ and $10 \sigma$, respectively. Middle panel: Same as in the left panel for HDCO. The magenta contours represent the low-velocity (LV; up to $-10 \mathrm{~km} \mathrm{~s}^{-1}$ with respect to $v_{\text {sys }}$ ) $\mathrm{SiO}(2-1)$ emission, tracing a large structure extending towards the south (up to 16 ", $4000 \mathrm{AU}$, from B1a), which creates the so-called "finger" feature (southern black triangle; Gueth et al. 1998) that coincides with the location of the oldest shock within $\mathrm{B} 1$. For $\mathrm{SiO}$ at $\mathrm{LV}$, the first contour and step are $5 \sigma\left(1 \sigma=50 \mathrm{mJy}\right.$ beam $\left.^{-1} \mathrm{~km} \mathrm{~s}^{-1}\right)$ and $10 \sigma$, respectively. Right panel: $\mathrm{p}-\mathrm{H}_{2} \mathrm{CO}\left(2_{0,2}-1_{0,1}\right)$ integrated emission (grey; also smoothed to the same beam of the $\mathrm{NH}_{2} \mathrm{CHO}$ image; Benedettini et al. 2013). The first contour and step are $3 \sigma\left(1 \sigma=3.3 \mathrm{mJy}^{-1} \mathrm{beam}^{-1} \mathrm{~km} \mathrm{~s}^{-1}\right)$ and $6 \sigma$, respectively. In colour is reported the $\mathrm{CH}_{3} \mathrm{CHO}\left(7_{0,7}-6_{0,6} \mathrm{E}+\mathrm{A}\right) / \mathrm{NH}_{2} \mathrm{CHO}$ $\left(4_{1,4}-3_{1,3}\right)$ line intensity ratio (derived where both emission is at least $3 \sigma$ ) smoothly decreasing from north to south (see the wedge).

The cosmic ray ionisation rate was previously constrained to be $\sim 3 \times 10^{-16} \mathrm{~s}^{-1}$ (Podio et al. 2014). The steady state abundances are then used as initial abundances for modelling the second step, with exceptions of the species that are injected into the gas phase because of the shock passage. In this second step, the density is set at $2 \times 10^{5} \mathrm{H}$-nuclei $\mathrm{cm}^{-3}$ and the temperature at $60 \mathrm{~K}$ (Lefloch et al. 2012; Gómez-Ruiz et al. 2015). The shock passage is accompanied by the sputtering of several species from the grain mantles into the gas phase, which corresponds to a sudden increase of their abundance. Again, following previous works, we increased the abundances of these gaseous species to simulate the sputtering. Table B.1 lists the species injected into the gas and their assumed abundances. The injected species have abundances similar to those measured by IR observations of the interstellar dust ices (Boogert et al. 2015). Specifically, they were constrained to match the abundances derived through direct observations of the 1100 years old L1157-B1a shock (Tafalla et al. 1995; Benedettini et al. 2013; Busquet et al. 2014). For some injected species, we chose the values derived by comparison of observations with model predictions. In addition, we slightly changed the values to fit the observed acetaldehyde and formamide abundances. The final model also reproduces the observed abundances of the species reported in Table B.1. When possible, we report the observed gas-phase abundances towards L1157-B1a, i.e. the youngest (1100 yr) shocked region within the B1 structure. Finally, we use a pure gas-phase model with no freeze-out included, since the involved timescale is too short for freeze-out to have any impact on the results. In the following, we 
Table B.1. Abundances (with respect to H-nuclei) of the species injected into the gas in the second step of the model and previously observed towards L1157-B1a.

\begin{tabular}{lcccc}
\hline \hline \multirow{2}{*}{ Species } & Injected (/H) & \multicolumn{2}{c}{ Observed (/H) } & Reference \\
& & Ices & L1157-B1 & \\
\hline $\mathrm{CO}_{2}$ & $3 \times 10^{-5}$ & $\leq 3 \times 10^{-4}$ & - & 1 \\
$\mathrm{H}_{2} \mathrm{O}$ & $2 \times 10^{-4}$ & $1.2 \times 10^{-4}$ & $1-3 \times 10^{-4}$ & 1,2 \\
$\mathrm{OCS}$ & $2 \times 10^{-6}$ & - & $\simeq 10^{-6 a}$ & 3 \\
$\mathrm{CH}_{3} \mathrm{OH}$ & $4 \times 10^{-6}$ & - & $5.5 \times 10^{-6 a}$ & 4 \\
$\mathrm{H}_{2} \mathrm{CO}$ & $1 \times 10^{-6}$ & - & $1.5 \times 10^{-6}$ & 4 \\
$\mathrm{NH}_{3}$ & $2 \times 10^{-5}$ & - & $>10^{-6}$ & 5 \\
$\mathrm{CH}_{3} \mathrm{CH}_{2}$ & $4 \times 10^{-8}$ & - & $2 \times 10^{-7 a}$ & 6 \\
\hline
\end{tabular}

Notes. ${ }^{(a)}$ These values have been indirectly derived by comparison of observations and model predictions.

References. 1. Boogert et al. (2015); 2. Busquet et al. (2014); 3. Podio et al. (2014); 4. Benedettini et al. (2013); 5. Tafalla \& Bachiller (1995); 6. Codella et al. (2015).

give details on the first two cases (the third one is a combination of both) discussed in the main text:

(1) Acetaldehyde and formamide are grain-surface chemistry products: the hypothesis is that both species are injected into the gas phase directly from the grain mantles, regardless of the mechanism that form these species there. Once in the gas, the two species undergo reactions that destroy them (Fig. 3). Specifically, both acetaldehyde and formamide are attacked by the most abundant gaseous ions, namely $\mathrm{H}_{3} \mathrm{O}^{+}, \mathrm{H}_{3}{ }^{+}$and $\mathrm{HCO}^{+}$, which produce protonated acetaldehyde and protonated formamide, respectively. Protonated formamide rapidly recombines with electrons and re-forms formamide in only $20 \%$ of cases, according to the KIDA database (formamide is not present in the UMIST database). Similarly, the recombination of protonated acetaldehyde produces acetaldehyde in only $9 \%$ of electron recombinations. While the rate and products of the protonated formamide recombination are guessed, those of acetaldehyde, from the UMIST database, are measured (Hamberg et al. 2010). However, since the two species are destroyed by the same ions, even if the branching ratios of the formamide recombination in the KIDA database are wrong, what matters is the percentage of electron recombinations that give back formamide, which is certainly not unity. In this respect, therefore, the result that formamide and acetaldehyde are not both grain-surface chemistry products is robust. (2) Acetaldehyde and formamide are gas-phase chemistry products: the hypothesis is that both species are produced by gasphase reactions after the injection into the gas of species previously frozen on the grain mantles. Only one reaction is known for the gas-phase formation of formamide, i.e. $\mathrm{NH}_{2}+\mathrm{H}_{2} \mathrm{CO} \rightarrow$ $\mathrm{NH}_{2} \mathrm{CHO}+\mathrm{H}$ (Barone et al. 2015; Vazart et al. 2016; Skouteris et al. 2017). The two mother species injected from the grain mantles to synthesise formamide are formaldehyde and ammonia. Both species have been detected in the solid state (Boogert et al. 2015) and are thought to be the result of hydrogenation on the grain surfaces of $\mathrm{CO}$ and $\mathrm{N}$, respectively. $\mathrm{NH}_{2}$ is then produced from ammonia via the reactions of $\mathrm{NH}_{3}$ with $\mathrm{H}_{3} \mathrm{O}^{+}$and $\mathrm{H}_{3}{ }^{+}$, which both give protonated ammonia $\mathrm{NH}_{4}{ }^{+}$. The electron recombination of $\mathrm{NH}_{4}{ }^{+}$then produces amidogen.

For acetaldehyde, a dozen reactions are listed in the KIDA and UMIST databases. Among these, the reaction $\mathrm{O}+\mathrm{CH}_{3} \mathrm{CH}_{2}$ $\rightarrow \mathrm{CH}_{3} \mathrm{CHO}+\mathrm{H}$ (Charnley et al. 1992; Harding et al. 2005; Yang et al. 2005) is the most efficient in the conditions appropriate for the L1157-B1 gas. Therefore, the two gaseous species necessary to synthesise acetaldehyde are atomic oxygen and ethyl radical. According to the astrochemical models, less than
$20 \%$ of gaseous oxygen is in the form of atomic oxygen. In L1157-B1, the $\mathrm{O}$ abundance is predicted to be $5 \times 10^{-6}$, in agreement with the bright [OI]-63 $\mu \mathrm{m}$ line observed (Benedettini et al. 2012) by Herschel in L1157-B1. The case of ethyl radical is a bit more complicated. It may be the result of the partial hydrogenation of $\mathrm{C}_{2} \mathrm{H}_{2}$ or $\mathrm{C}_{2} \mathrm{H}_{4}$ on the grain surfaces and may be directly sputtered from the grain mantles as such; we note that no observations exist about the abundance of this species in the solid form and no computations have been carried out. On the other hand, the ethyl radical can be produced starting from ethane, which, in turn, is formed by the total hydrogenation of $\mathrm{C}_{2} \mathrm{H}_{2}$ or $\mathrm{C}_{2} \mathrm{H}_{4}$ on the ice before sputtering. We ran, therefore, a case where only ethane is liberated into the gas phase; even assuming an injection of $4 \times 10^{-6}$ ethane, namely $\sim 10 \%$ of $\mathrm{CO}$, the predicted acetaldehyde abundance remains 10 times lower than the observed acetaldehyde abundance. Therefore, ethyl radical needs to be directly injected into the gas phase from the grain mantles. As discussed in the main text, an abundance of $4 \times 10^{-8}$ is necessary to reproduce the L1157-B1 observations. Assuming an ethane abundance of $4 \times 10^{-6}$, this would imply that about $1 \%$ of it is liberated from the grain mantles as the partially hydrogenated "cousin" ethyl radical. Alternatively, it is possible that the full hydrogenation leading to ethane is not very efficient on the grain surfaces.

In order to test the robustness of the results showed in Fig. 3 and discussed in the main text, we also ran a case where $1 \%$ of ammonia is directly injected as amidogen (namely $2 \times 10^{-7}$ ), which is possibly analogous to the ethyl radical. In this case, we obtain almost exactly the same results shown in Fig. 3, with differences of a few \% within the first $2000 \mathrm{yr}$, confirming that the important mother species in the formamide formation is indeed ammonia. To summarise, the comparison between the observations and the model predictions leads to ammonia and ethyl radical as being the two needed previously frozen mother species of acetaldehyde and formamide, respectively, with frozen-andinjected amidogen and ethane being minor actors.

Finally, it is possible that before equilibrating at $60 \mathrm{~K}$, the shocked gas passed through a high-temperature period. In order to verify whether this period would affect the results reported in Fig. 3 and our conclusions, we ran two models with the gas temperature equal to $1000 \mathrm{~K}$ in case (1) and case (2). We found (1) acetaldehyde and formamide are grain-surface chemistry products: during the first $2000 \mathrm{yr}$ of a possible hightemperature period the predicted abundance ratio of acetaldehyde and formamide remains practically the same, as they are destroyed by the same molecular ions $\left(\mathrm{H}_{3} \mathrm{O}^{+}, \mathrm{H}_{3}{ }^{+}\right.$, and $\left.\mathrm{HCO}^{+}\right)$, such that it does not affect the output of Fig. 3 and our conclusion that this case does not reproduce the observed behaviour. (2) Acetaldehyde and formamide are gas-phase chemistry products: at $1000 \mathrm{~K}$, the formamide rate of formation in the gas is very low, as it decreases with a power of 2.56 in temperature (Vazart et al. 2016), such that no formamide is appreciably synthesised during the high-temperature period. On the contrary, the predicted acetaldehyde abundance is almost the same as that at $60 \mathrm{~K}$ during the first $2000 \mathrm{yr}$. Therefore, a high-temperature period preceding the $60 \mathrm{~K}$ period would lean towards the same direction of our conclusions. The region with only acetaldehyde is younger (and possibly also warmer), while the region with formamide identifies an older shocked region and formamide is synthesised via the gas-phase reaction $\mathrm{NH}_{2}+\mathrm{H}_{2} \mathrm{CO}$. In summary, even if a hightemperature period, not included in our simple model, preceded the present $60 \mathrm{~K}$ equilibrated gas temperature, the effects would not change our main conclusion, namely that formamide has to be a gas chemistry product. 